hep-ph/0207003

\title{
Black hole evaporation with separated fermions
}

\author{
Tao Han, Graham D. Kribs, and Bob McElrath \\ Department of Physics, University of Wisconsin, Madison, WI 53706 \\ than@pheno.physics.wisc.edu,kribs@physics.wisc.edu,rsmcelrath@wisc.edu
}

\begin{abstract}
In models with a low quantum gravity scale, a well-motivated reason to expect quark and lepton fields are localized but physically separated is to avoid proton decay. This could happen in a "fatbrane" or in an additional, orthogonal $1 / \mathrm{TeV}$ sized dimension in which the gauge and Higgs fields live throughout. Black holes with masses of order the quantum gravity scale are therefore expected to evaporate non-universally, preferentially radiating directly into quarks or leptons but not both. Should black holes be copiously produced at a future hadron collider, we find the ratio of final state jets to charged leptons to photons is 113:8:1, which differs from previous analyses that assumed all standard model fields live at the same point in the extra dimensional space.
\end{abstract}

Black holes are the most captivating prediction of general relativity despite the dearth of experimental evidence to date. Testing black hole creation and evaporation in particle physics experiments has been considered, until recently, far out-of-reach due to the feebleness of gravitational interactions when compared with standard model forces. If space has many compact extra dimensions, however, the fundamental quantum gravity scale $M_{*}$ might be as low as a TeV [1]. Should we be so lucky, future high energy colliders [2, 3] and ultra-high energy cosmic-ray collisions with the atmosphere and the earth's crust [4] can be production sources of black holes. The black holes then quickly decay through Hawking radiation [5] on collider time scales, emitting energetic jets, leptons, photons, and neutrinos.

Previous analyses have generally assumed that baryon number $B$ and lepton number $L$, or more precisely $B-L$, is conserved in the evaporation spectrum. The conservation of $B-L$ must hold to extremely high accuracy to prevent the proton from decaying through operators suppressed by the lowered quantum gravity scale, of order a $\mathrm{TeV}$. However, in large extra dimension scenarios there is no reason for $B-L$ to be conserved; additional assumptions must augment the absence of a desert. One approach is to use discrete gauge symmetries [6, 7], analogous to imposing matter parity in low energy supersymmetric models. Alternatively, there is an intrinsically extra dimensional proposal that physically separates the quark and lepton fields far enough so that their wavefunction overlap is exponentially suppressed [8].

Consider a very massive black hole, with a mass much larger than $M_{*}$. Initially, it is so large as to "fill up" all compact extra dimensions. As the black hole evaporation proceeds, the mass decreases, the temperature rises, and the horizon shrinks. Eventually, the black hole horizon will become smaller than the large extra dimensions in which only gravity lives ("gravity-only" dimensions). At this point the black hole is radiating mostly into brane modes, for reasons that were first clearly elucidated in [9]. Roughly speaking, the naive enhancement that one might guess of radiation into a tower of Kaluza-Klein
(KK) modes is compensated for by the small overlap of the higher dimensional field's wavefunction with the black hole horizon. More detailed calculations confirm this intuition [9], which we apply to our scenario.

Since there are many more standard model degrees of freedom on the brane over the pristine gravity-only bulk, the overwhelmingly dominant evaporation products are brane modes. Once the black hole horizon shrinks to become smaller than the separation between quarks and leptons, of order 10 to 100 times the inverse quantum gravity scale, a qualitative change is expected in the black hole evaporation process. Black holes stuck to the brane where quarks live will evaporate directly into quarks but not leptons, while black holes stuck to the brane in which leptons live will evaporate directly to leptons but not quarks. This observation has dramatic consequences for collider and cosmic-ray signals of black holes.

In a scenario with large extra dimensions, spacetime consists of our ordinary three space plus one time dimensions plus several additional curled-up space dimensions compactified in a volume $V_{D}$. For a torus, for example, $V_{D}=(2 \pi R)^{D-4}$. The higher dimensional Newton's constant $G_{D}$ is related to the four dimensional one $G_{4}$ through $G_{D}=G_{4} V_{D}$. The coupling of a 4-dimensional graviton to matter is suppressed by the reduced Planck scale

$$
M_{\mathrm{Pl}} \equiv \frac{1}{\sqrt{8 \pi G_{4}}} \simeq 2 \times 10^{18} \mathrm{GeV}
$$

which is the scale where quantum gravity effects are expected to be order one. The coupling of a higher dimensional graviton to higher dimensional matter is analogously suppressed by

$$
M_{*} \equiv\left(\frac{1}{8 \pi G_{D}}\right)^{1 /(D-2)}
$$

which we take to be our definition of the quantum gravity scale. In a moment it will be clear that this definition is physically well-motivated using considerations of black hole dynamics. 
The basic properties of black holes in higher dimensions are by now well understood. We will be exclusively concerned with black holes near the end of their life when their mass $M_{\mathrm{bh}}$ is larger than $M_{*}$ but their horizon $r_{h}$ is smaller than the size of any compact dimension. The reasons for this are twofold: It is in this regime that the evaporation is expected to be non-universal, and secondly future collider or cosmic-ray production is expected to create black holes whose mass is not too much larger than $M_{*}$. In all analyses below we treat black holes as semi-classical $D$-dimensional objects. We briefly outline their salient properties. The horizon of a $D$-dimensional black hole is given by 10

$$
r_{h}=\frac{1}{M_{*}}\left(\frac{M_{\mathrm{bh}}}{M_{*}}\right)^{1 /(D-3)}\left(\frac{2}{(D-2) \Omega_{D-2}}\right)^{1 /(D-3)}
$$

where

$$
\Omega_{D} \equiv \frac{2 \pi^{(D+1) / 2}}{\Gamma[(D+1) / 2]}
$$

is the volume of a unit $D$-sphere. The $D$-dependent numerical factor on the far right-hand side of (3) varies from about $1 / 4$ to $1 / 2$ for $6 \leq D \leq 11$. The black hole temperature is simply

$$
T=\frac{D-3}{4 \pi r_{h}}
$$

that is well approximated (to within $\pm 15 \%$ ) by

$$
T \simeq M_{*}\left(\frac{M_{\mathrm{bh}}}{M_{*}}\right)^{-1 /(D-3)}
$$

for any number of dimensions $4 \leq D \leq 11$. Interestingly, in four dimensions the temperature is exactly equal to the reduced Planck scale $T=M_{\mathrm{Pl}}$ for a black hole with mass $M_{\mathrm{bh}}=M_{\mathrm{Pl}}$. Similarly, with our definition of $M_{*}$, a higher dimensional black hole has a temperature almost identical to $M_{*}$ for a black hole with mass $M_{\mathrm{bh}}=M_{*}$. No other choice of definition of the higher dimensional Planck scale has this property.

We imagine that quarks and leptons are branelocalized fields with the smallest physically reasonable Gaussian width $\sim 1 / M_{*}$. Quarks live on a "quark brane", while leptons live on a "lepton brane", physically separated by a distance $L \sim(10-100) / M_{*}$, where the uncertainty in the separation length depends on the nature of the induced proton decay operators. We shall optimistically assume $L \sim 10 / M_{*}$ is sufficient to suppress dimension-6 proton decay operators such as $q q q \mathrm{l} / M_{*}^{2}$, which should be correct upon integrating out all but the most bizarre (wormhole-like) field configurations. Gauge and Higgs fields must propagate in the bulk so that quarks and leptons interact with one another just as in the Standard Model. Of course gauge and Higgs fields will also have a KK tower of excitations with masses
$M_{K K}=L^{-1}, 2 L^{-1}$, etc., with couplings that may differ significantly from one-brane-localized models 11]. In extra dimensional models with bulk gauge and Higgs fields but localized matter fermions, there are strong experimental constraints on the mass of the lightest KK gauge boson from precision electroweak measurements (see, e.g., 12 ). Although no analysis has been done for this particular scenario, it is quite reasonable to expect bounds similar to those found for all matter stuck to the same point, namely $M_{K K} \gtrsim 2-5 \mathrm{TeV}$. This means that the scale of quantum gravity $M_{*} \sim 20-50 \mathrm{TeV}$.

This is a somewhat large scale requiring fine-tuning of the Higgs mass to one part in about a thousand. However, a larger $M_{*}$ has several well-known advantages. Generic four-fermi operators suppressed by $1 / \mathrm{TeV}$ give rise to large contributions to flavor-changing neutral currents, $\mathrm{CP}$ violating phenomena, etc., that cannot be forbidden by discrete (gauged) symmetries. Here, with $M_{*}$ in the tens of $\mathrm{TeV}$ region, these dangerous operators are naturally suppressed with order one coefficients. For example, a recent analysis of the constraint arising from neutron-anti-neutron oscillations suggests $M_{*} \gtrsim 45 \mathrm{TeV}$ [13], roughly in alignment with the scale in our scenario. Another advantage of a larger scale for quantum gravity is that it allows one fewer gravity-only extra dimensions. The constraints on two extra dimensions from Supernova cooling [14] suggest $M_{*}$ should be larger than tens of $\mathrm{TeV}$, but this is automatically satisfied in our setup. We are therefore content with two or more gravity-only extra dimensions that open up at long distances (up to $0.1 \mathrm{~mm}$ or so), and one extra dimension with gauge and Higgs fields propagating in the bulk to open up around a few $\mathrm{TeV}$. The resulting volume of compactified space is arranged such that $M_{*}$ is of order $20-50 \mathrm{TeV}$.

Black hole formation can occur astrophysically, such as from primordial density fluctuations in the early universe, through high-energy cosmic-ray collisions, or at a sufficiently high energy collider. There are by now several arguments suggesting black hole formation can occur at colliders [2, 3, 15] (but for opposing views see 16]). The arguments in favor of black hole formation are persuasive, but we emphasize that the evaporation spectrum is modified near the end of a black hole's life no matter how it was formed.

The decay of a thermalized black hole proceeds through Hawking radiation [5]. The evaporation rate of a particle species $i$ of spin $s$ is given by the usual blackbody spectrum

$$
\frac{d N_{i}}{d t}=\frac{c_{i} \sigma_{s}}{e^{E / T}-(-1)^{2 s}} \frac{d^{n-1} k}{(2 \pi)^{n-1}} .
$$

where $k$ is the $(n-1)$-momenta of the particle living in $n$ dimensions, $c_{i}$ is the multiplicity of the species, and $\sigma_{s}$ is the absorption cross section often simply referred to as the greybody factor. Note that the number of dimensions, $n$, in which a particular standard model field lives 
should not be confused with dimensionality of spacetime $D$. We find it convenient to rewrite the greybody factor as a dimensionless constant normalized to the black hole surface area $A_{n}$ seen by the $n$-dimensional fields,

$$
\Gamma_{s}=\sigma_{s} / A_{n}
$$

For four-dimensional fields, the emission rate is simply 117

$$
\frac{d N_{i}}{d E d t}=\frac{A_{4}}{8 \pi^{2}} \frac{c_{i} \Gamma_{s} E^{2}}{e^{E / T}-(-1)^{2 s}} .
$$

The greybody factor $\Gamma_{s}$ is in general both spin- and energy-dependent. Greybody factors were first calculated by Page 17] for four-dimensional black holes. Higher dimensional greybody factors have not been calculated except for scalars 18. As was emphasized in [17, 19, one needs the full energy dependence of the greybody factor to calculate the full emission spectrum. However, the integrated power emission is reasonably well approximated by taking the high energy limit of $\Gamma_{s}$, the geometric optics approximation. A black hole acts as a perfect absorber of a slightly larger radius, $r_{c}$, given in $D$ dimensions by [9]

$$
r_{c}=\left(\frac{D-1}{2}\right)^{1 /(D-3)} \sqrt{\frac{D-1}{D-3}} r_{h}
$$

We shall employ this approximation to compute the particle emission and energy spectra in our setup. The one final refinement that we incorporate is to correct for the differing power into spin-0, spin- $1 / 2$, and spin- 1 modes known in four dimensions by integrating the spectra numerically 17. This results in a suppression of power into spin-1/2 and spin-1 modes parameterized by $\Gamma_{s}$

$$
\Gamma_{s=0}=1 \quad \Gamma_{s=1 / 2} \simeq 2 / 3 \quad \Gamma_{s=1} \simeq 1 / 4 .
$$

Once higher dimensional energy-dependent greybody factors become available, they could be easily incorporated here, although we do not expect any qualitative changes to our results.

The area of a black hole in the geometric optics approximation is

$$
A_{n}=\Omega_{n-2}\left(\frac{D-1}{2}\right)^{(n-2) /(D-3)}\left(\frac{D-1}{D-3}\right)^{(n-2) / 2} r^{n-2} .
$$

The particle emission spectrum into brane fermions is therefore

$$
\frac{d N_{i}}{d E d t}=\frac{c_{i} \Gamma_{s} A_{4}}{8 \pi^{2}} \frac{E^{2}}{e^{E / T}+1} .
$$

and into bulk gauge bosons and Higgs is

$$
\frac{d N_{i}}{d E d t}=\frac{c_{i} \Gamma_{s} A_{n} \Omega_{n-3}}{(n-2)(2 \pi)^{n-1}} \frac{E^{n-2}}{e^{E / T}-1} .
$$

The flux emission spectra can be easily integrated, and we find

$$
\frac{d N_{i}}{d t}=\frac{c_{i} \Gamma_{s} \Omega_{n-3} A_{n} f}{(2 \pi)^{n-1}(n-2)}\left(\frac{D-3}{4 \pi r_{h}}\right)^{n-2} \Gamma(n-1) \zeta(n-1)
$$

where $f=1$ for bosons and $f=1-2^{2-n}$ for fermions. For our setup, the ratio of the emitted flux into a single brane field over a single bulk field is

$$
\frac{d N_{\text {brane }} / d t}{d N_{\text {bulk }} / d t} \simeq 2.2 \frac{c_{\text {brane }} \Gamma_{\text {brane }}}{c_{\text {bulk }} \Gamma_{\text {bulk }}} .
$$

The numerical factor 2.2 corresponds to a $(D, n)=(7,5)$. Varying the dimensionality $7 \leq D \leq 11$ and $5 \leq n \leq$ $D-2$ one finds the numerical factor ranges from 1.4 to 3.5. Hence, our flux estimates should be valid to within a factor of 1.5 for a wide range of models.

The direct emission rate is now straightforward to calculate. For a black hole in $D=7$ dimensions with gauge/Higgs fields in $n=5$ dimensions, we simply use Eq. (11) and the degrees of freedom per species

$$
\begin{gathered}
c_{q}=72 ; \quad c_{\ell}=24 ; \quad c_{g}=24 ; \quad c_{\gamma}=3 ; \quad c_{h}=1 ; \\
c_{W^{ \pm}, Z}=9 ; \quad c_{G^{ \pm}, G^{0}}=3 ;
\end{gathered}
$$

to obtain the following relative direct particle emission rates for a black hole whose horizon size is smaller than the separation distance between quarks and leptons. With our formalism there is no sum over the KK states of five dimensional fields; this is fully accounted for in the particle multiplicities and the $n$-dimensional flux emission rate (15). For the neutrino multiplicity, both leftand right-handed neutrinos were included. In five dimensions each gauge boson has three physical polarizations, and the bulk Goldstone bosons can be absorbed in the $W^{ \pm}, Z$ multiplicity $c_{W^{ \pm}, Z}^{\mathrm{eff}}=c_{W^{ \pm}, Z}+c_{G^{ \pm}, G^{0}} \Gamma_{0} / \Gamma_{1} \simeq 21$. Assuming a black hole is formed on the quark or lepton brane and does not wander off (this is unlikely for both energetic [9] and kinematic reasons), we find

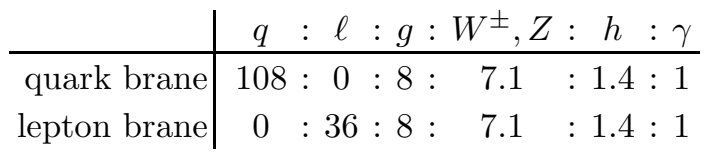

Accounting for gauge boson decay and top quark decay, then associating each quark or gluon as a single jet, we can estimate the final jet to charged lepton to neutrino (plus graviton) to photon ratio,



This leads to very striking consequences for black hole production and evaporation. At a hadron collider, the types of partonic collisions include quark-on-quark, quark-on-gluon, or gluon-on-gluon. The first two types 
of partonic collisions produce a black hole on the quark brane that evaporates into the final state particles as specified above. Gluon fusion, however, could naively create a black hole anywhere in the extra dimension(s) in which gluons can propagate. The evaporation spectrum is likely to be quite unusual, dominantly into bulk modes only. However, gluon fusion is not expected to be a dominant production process due to the relatively low gluon luminosity for producing a very heavy object. For illustration, consider a VLHC with a center-of-mass energy of $200 \mathrm{TeV}$. The production cross section for $M_{\mathrm{bh}}=50$ $\mathrm{TeV}$ for $D=11$ from $q \bar{q}$ annihilation is about $15-110 \mathrm{fb}$ for $M_{*}=20-50 \mathrm{TeV}$, leading to a few thousand events annually. We have argued that $M_{*}$ must be at least in the tens of $\mathrm{TeV}$ region in our setup, and so the LHC and lepton colliders are not energetic enough to produce black holes, although they could find the KK excitations of gauge/Higgs bosons. (Collisions of heavy nuclei might also be of interest 20].)

Black holes could also be produced through high energy cosmic-ray collisions. If the primary incident particle is a proton, one would expect hadronic showers to dominate; conversely if the primary is a neutrino, there would be a large fraction of energetic charged leptons. Black hole production from neutrino collisions, however, proceed only through $\nu$ g interactions that are significant only for lighter black holes and at higher energies. Typically, the cross section for $M_{b h}=50 \mathrm{TeV}$ for $D=11$ is about $0.1-1 \mathrm{pb}$ at $E_{\nu} \simeq 10^{19} \mathrm{eV}$.

In summary, we have found that in models with large extra dimensions and separated fermions the evaporation spectrum of black holes significantly changed once the black hole horizon is smaller than the separation distanc between quarks and leptons. For example, our estimates suggest that black holes produced at hadron colliders in this regime will emit a large multiplicity of jets over leptons or photons. While we have concentrated on the broad characteristics of black hole evaporation with separated fermions, there are inevitably several improvements that could be done to further refine our predictions; we list a few of the more important ones: Throughout this analysis we have neglected the angular momentum of the black hole. In general, as argued in [2, 21], black holes formed in colliders or cosmic-ray collisions are very likely to have significant angular momentum, and so accounting for the spin-down phase is quite important to accurately predict the energy and angular distributions of the black hole emission. Another improvement is to calculate the energy-dependent greybody factors for arbitrery spacetime dimensionality. Another is to account for the backrean of high energy particle emission on the black hole geometry; in particular, accounting for the deviations from a thermal spectrum. Finally, new stringy dynamics could alter the very final evaporation spectrum 222 depending on the relative hierarchy between $M_{*}$ and the string scale. With evidence for black holes in hand, we may well learn the spacetime structure and standard model field geography through a detailed analysis of particle counting and energy spectra resulting from black hole evaporation.

We thank J. Hewett, N. Kaloper, and S. Thomas for encouragement and discussions. GDK thanks the SLAC theory group and ITP Santa Barbara for their generous hospitality where this work was begun. This work was supported in part by the U.S. Department of Energy under contract DE-FG02-95ER40896 and in part by the Wisconsin Alumni Research Foundation.

[1] N. Arkani-Hamed, S. Dimopoulos and G. R. Dvali, Phys. Lett. B 429, 263 (1998) hep-ph/9803315.

[2] S. B. Giddings and S. Thomas, Phys. Rev. D 65, 056010 (2002) hep-ph/0106219.

[3] S. Dimopoulos and G. Landsberg, Phys. Rev. Lett. 87, 161602 (2001) hep-ph/0106295.

[4] J. L. Feng and A. D. Shapere, Phys. Rev. Lett. 88, 021303 (2002) hep-ph/0109106; R. Emparan, M. Masip and R. Rattazzi, Phys. Rev. D 65, 064023 (2002) hepph/0109287; A. Ringwald and H. Tu, Phys. Lett. B 525, 135 (2002) hep-ph/0111042. L. A. Anchordoqui, J. L. Feng, H. Goldberg and A. D. Shapere, hepph/0112247; M. Kowalski, A. Ringwald and H. Tu, Phys. Lett. B 529, 1 (2002) hep-ph/0201139. J. AlvarezMuniz, J. L. Feng, F. Halzen, T. Han and D. Hooper, hep-ph/0202081.

[5] S. W. Hawking, Commun. Math. Phys. 43, 199 (1975).

[6] L. M. Krauss and F. Wilczek, Phys. Rev. Lett. 62, 1221 (1989).

[7] I. Antoniadis, N. Arkani-Hamed, S. Dimopoulos and G. R. Dvali, Phys. Lett. B 436, 257 (1998) hepph/9804398]; N. Arkani-Hamed and S. Dimopoulos, Phys. Rev. D 65, 052003 (2002) hep-ph/9811353.

[8] N. Arkani-Hamed and M. Schmaltz, Phys. Rev. D 61, 033005 (2000) hep-ph/9903417.

[9] R. Emparan, G. T. Horowitz and R. C. Myers Phys. Rev. Lett. 85, 499 (2000) hep-th/0003118

[10] R. C. Myers and M. J. Perry, Annals Phys. 172, 304 (1986).

[11] N. Arkani-Hamed, Y. Grossman and M. Schmaltz, Phys. Rev. D 61, 115004 (2000) hep-ph/9909411.

[12] T. G. Rizzo and J. D. Wells, Phys. Rev. D 61, 016007 (2000) hep-ph/9906234; A. Delgado, A. Pomarol and M. Quiros, JHEP 0001, 030 (2000) hep-ph/9911252

[13] S. Nussinov and R. Shrock, Phys. Rev. Lett. 88, 171601 (2002) hep-ph/0112337.

[14] S. Cullen and M. Perelstein, Phys. Rev. Lett. 83, 268 (1999) hep-ph/9903422 ; V. D. Barger, T. Han, C. Kao and R. J. Zhang, Phys. Lett. B 461, 34 (1999) hepph/9905474]; C. Hanhart, D. R. Phillips, S. Reddy and M. J. Savage, Nucl. Phys. B 595, 335 (2001) nuclth/0007016

[15] D. M. Eardley and S. B. Giddings, gr-qc/0201034; T. G. Rizzo, JHEP 0202, 011 (2002) hep-ph/0201228; S. D. Hsu, hep-ph/0203154 A. Jevicki and J. Thaler, hep-th/0203172.

[16] M. B. Voloshin, Phys. Lett. B 518, 137 (2001) hep- 
ph/0107119]: M. B. Voloshin, Phys. Lett. B 524, 376 (2002) hep-ph/0111099.

[17] D. N. Page, Phys. Rev. D 13, 198 (1976).

[18] P. Kanti and J. March-Russell, hep-ph/0203223.

[19] J. H. MacGibbon and B. R. Webber, Phys. Rev. D 41, 3052 (1990).

[20] A. Chamblin and G. C. Nayak, hep-ph/0206060.

[21] A. V. Kotwal and C. Hays, hep-ph/0206055.
[22] S. Dimopoulos and R. Emparan, Phys. Lett. B 526 , 393 (2002) hep-ph/0108060; E. J. Ahn, M. Cavaglia and A. V. Olinto, hep-th/0201042 L. A. Anchordoqui, J. L. Feng and H. Goldberg, Phys. Lett. B 535, 302 (2002) hep-ph/0202124; K. Cheung, hep-ph/0205033. K. Cheung and C. H. Chou, hep-ph/0205284. 